\title{
Gastrointestinal Symptoms in Endometriosis
}

\author{
SHAKEELA ISHRAT ${ }^{1}$ FARZANA DEEBA ${ }^{2}$, PARVEEN FATIMA ${ }^{3}$
}

\begin{abstract}
:
Background: Endometriosis is frequently associated with gastrointestinal symptoms, in addition to dysmenorrhoea, dyspareunia and infertility. Gastrointestinal tract is not always involved when gastrointestinal symptoms are present.

Method: A cross sectional study was conducted in the department of Obstetrics \& Gynaecology of Bangladesh Sheikh Mujib Medical University (BSMMU) on 55 infertile women with surgical diagnosis of endometriosis with the objective to determine the frequency of gastrointestinal symptoms in our population of women with endometriosis and to find out whether they were relevant to severity of endometriosis.

Results: There was a high prevalence (37.5\%) of gastrointestinal symptoms in women with endometriosis. Bloating was the most frequent gastrointestinal symptom. Among the women with severe stage IV endometriosis, $57.6 \%$ had gastrointestinal symptoms.

Conclusion: A gynecologist must consider the gastrointestinal symptoms while dealing with the women with endometriosis. Further evaluation of gastrointestinal tract involvement is necessary for counseling and comprehensive management of the women with endometriosis.
\end{abstract}

Key words: Endometriosis, gastrointestinal symptoms

\section{Introduction:}

Endometriosis is the proliferation of endometrial tissue outside endometrial cavity. It is one of the most common benign gynaecological disorders. The disease is present in about $6-10 \%$ of all reproductive aged women and its prevalence ranges from 25 to $35 \%$ in infertile women. ${ }^{1}$

Endometriosis can remain asymptomatic, but often there are dysmenorrhoea, dyspareunia and infertility associated with it. The heterogenous presentation and investigations have poor predictive value for diagnosis of endometriosis in women. Gold standard for diagnosis is visual inspection of lesions on laparoscopy or laparotomy.

Gastrointestinal tract is involved in approximately $5 \%$ (3.8-3.7\%) of patients with endometriosis. ${ }^{2}$ The most frequently involved organ is sigmoid colon followed by rectum, ileum, appendix and caecum. Large endometriotic nodules involving bowel cause pain and a large variety of gastrointestinal symptoms like diarrhoea, constipation and abdominal bloating.
Cyclical rectal bleeding or hematochezia is rarely observed when the mucosa is infiltrated by endometriosis. But the frequency of gastrointestinal symptoms in women with endometriosis cannot always be explained by involvement of bowel.

The objective of our study was detailed exploration of clinical presentations to assess the frequency and relevance of gastrointestinal symptoms in endometriosis.

\section{Method:}

This cross sectional study was conducted in the departments of Obs \& Gyane of BSMMU from $1^{\text {st }}$ January 2017 to $31^{\text {st }}$ December 2017.

We studied the symptoms in a consecutive series of 55 women with surgical diagnosis of endometriosis. All the women had laparotomy or laparoscopy for visual confirmation of diagnosis. The revised American Fertility Society Endometriosis scores were used for staging of endometriosis during surgery. A structured questionnaire was completed with

1. Associate Professor, Dept of Obs \& Gynae, BSMMU, Dhaka

2. Associate Professor, Infertility unit, Dept of Obs \& Gynae, BSMMU, Dhaka

3. Professor \& Chairman, Dept of Obs \& Gynae, BSMMU, Dhaka

Address of Correspondence: Dr Shakeela Ishrat, Associate Professor, Dept. of Obs \& Gynae, BSMMU, Dhaka, Cell phone: 01729897221, E-mail: shakeelaishrat@yahoo.com 
assistance from the women, documenting the symptoms, both gynaecological and gastrointestinal. Data was analyzed using SPSS 18.0 for windows. Ethical Changes was obtained from the institution written informed consent was taken from the patients: confidentiality was strictly maintained.

\section{Results:}

Fifty five consecutive patients with surgical diagnosis of endometriosis were studied. Mean age was 27.84 yrs (+ 5.96 standard deviation), range 16-43 yrs. Unmarried women were $5.2 \%, 60.3 \%$ had primary infertility, 3.5\% had secondary infertility. Most women $(91.45 \%)$ had dysmenorrhoea (Table I). There was high prevalence $(37.9 \%)$ of gastrointestinal symptoms in women with endometriosis. Bloating was the most frequent gastrointestinal symptom (Table II).

Stage IV endometriosis was associated with bowel adhesion. But gastrointestinal symptoms are present in $57.6 \%$ of women with stage IV endometriosis. It can be inferred that gastrointestinal symptoms are not always associated with bowel adhesion.

Table-I

Gynaecological symptoms in women with endometriosis $(n=55)$

\begin{tabular}{lc}
\hline Gynaecological symptoms & Percentage \\
\hline Dysmenorrhea & 91.4 \\
Dyspareunia & 36.2 \\
Chronic pelvic pain & 37.9 \\
\hline
\end{tabular}

Table-II

Gastrointestinal symptoms in women with endometriosis $(n=55)$

\begin{tabular}{lc}
\hline Gastrointestinal symptoms & Percentage \\
\hline Bloating & 13.8 \\
Crampy abdominal pain & 20.7 \\
Change in bowel habits & 12.1 \\
Decreased bowel movement & 10.3 \\
Hematocezia & 5.2 \\
Anorexia & 12.1 \\
\hline
\end{tabular}

\section{Discussion:}

The objective of the study was to explore the relevance of gastrointestinal symptoms to endometriosis. We found high prevalence (37.9\%) of gastrointestinal symptoms among women with endometriosis. The presence of gastrointestinal symptoms are not always associated with bowel adhesion.

In a similar study ${ }^{3}$ which systemically explored the symptoms in a consecutive series of 355 women with endometriosis, ninety percent of women had gastrointestinal symptoms of which bloating was most common (83\%). Bowel lesion was present only on $76 \%$.

A case cohort study ${ }^{4}$ reveals that majority of endometriosis patients experience more severe gastrointestinal symptoms than controls. Localization of endometriosis had no association with symptoms, except lesions within and close to bowel.

The frequent presence of gastrointestinal symptoms in women with endometriosis can be explained in different ways.

One third of otherwise asymptomatic women may experience gastrointestinal symptoms at the time of menstruation ${ }^{5}$. Almost $50 \%$ of women with functional bowel disorders report an increase in symptoms during menstruation ${ }^{5}$. Women who suffer from dysmenorrhea are more likely to have functional bowel disorder. Prostaglandins released from uterus at the time of menstruation may cause diarrhoea.

Bowel is involved in about $5 \%$ of patients with endometriosis ${ }^{2}$. The most frequent location is sigmoid colon followed by rectum, ileum, appendix and caecum. Large endometriotic nodules involving bowel cause pain and gastrointestinal symptoms like diarroea, constipation and abdominal bloating.

Depending on the depth and infiltration and accompanying symptoms, resection of affected bowel may be necessary, even when both ovaries are being removed ${ }^{7}$. An sclerosis and fibrotic reaction associated with the lesion may lead to persistence of the symptoms, such as constipation.

Irritable bowel syndrome is an intestinal disorder characterized by abdominal pain and discomfort associated with changes in bowel habits in the absence of organic disease. ${ }^{1}$

Women with endometriosis has higher rate of pain symptoms associated with peripheral and central sensitization such as irritable bowel syndrome. Women with chronic pelvic pain who have endometriosis may be prone to depression, anxiety and chronic fatigue ${ }^{8}$. 
The study of Matteus $\mathrm{JR}^{7}$ et al reveals a consistent and distinct abnormality of bowel function in women with endometriosis. There is an abnormal motility pattern. Ampulla of Vater and duodenal wall spasm may be the result of injury to and lack of inhibitory control of intrinsic nervous system. The inhibitory neural circuits are the noradrenergic, non cholinergic inhibitory system. The neural transmitter is nitric oxide and/or post ganglionic neurons from the spinal cord and the neural transmitters as norepinephrineepinephrine.

During evaluation of a patient with endometriosis , the clinicians should spend some minutes investigating bowel habits. Whenever presence of bowel endometriosis is suspected, an imaging technique ( TVS, rectal endoscopic USG, double contrast barium enema, MRI) should be used to evaluate possible diagnosis. Once the diagnosis of bowel endometriosis is suspected, counseling is mandatory and options available are expectant management, medical treatment and surgery.

Laparoscopy is the gold standard for diagnosis of endometriosis but evaluation of the extent of deeply infiltrating endometriosis can be extremely difficult and misleading. On the other hand microscopic implants may be missed. Clinical examination, preferably during menstruation, is an essential clinical tool to diagnose deep endometriosis and a scarred cul de sac.

Improvement of symptoms by radical excision of all lesions is reported by several authors ${ }^{(2,7-8)}$. Removal of all endometriotic lesions is mandatory to obtain an optimum relief of symptoms. The observed associations between gastrointestinal symptoms and endometriosis could be evaluated further by follow up of the changes or improvement following adhesiolysis.

\section{Conclusion:}

A gynaecologist must investigate bowel function during the evaluation of a woman with endometriosis. Further studies should include a diagnosis or an exclusion of IBS in women with endometriosis. Further research is needed to explore what common hormonal or neurologic pathways may underlie the coexistence of menstrual and gastrointestinal symptoms.

\section{References:}

1. Sarajari S, Muse KN, Fox MD. Endometriosis. In: DeCherny AH, Laufer N,editors. Current diagnosis and treatment : Obstetrics \& Gynaecology. $11^{\text {th }}$ ed, United States: McGraw Hill; 2013 ; 911-919

2. RemargidaV, Ferriro S, Fulcheri E, Ragni N, Martia DC. Bowel endometriosis presentation, diagnosis and treatment. Obstetrical and Gynaecological Survey 2007; 62(7):461-470

3. Maroun P, Cooper MJW, Reid GD, Keirse MJNN. Relevance of gastrointestinal symptoms in endometriosis. ANZ Journal of Obstet \& Gyaecol 2009; $49: 411-414$

4. Malin EK, Roth B, Ekstrom P, Valentin L, Bingtsson M, Ohlsson B. Gastrointestinal symptoms among endometriosis patients-A case-cohort study. BMC Woman's Health 2015; 15:59

5. Moore J, Barlow D, Jewell D, Kennedy S. Do gastrointestinal symptoms vary with the menstrual cycle? BCOG 1998;105:322-25

6. Heitemper MM, Chang Li. Do fluctuations in ovarian hormones affect gastrointestinal symptoms in women with irritable bowel syndrome? Gend Med 2009; 6(2): 152-162

7. Mattheus JR, Franklin R, Quast DL, Fraga N, Loften C, Yates L, Harrison V. Relation of endometriosis and neuromuscular disease of gastrointestinal tract: new analysis. Fertil Steril 1998; 70(1):81-88

8. Hartmeyers P, Ceelen W, Gillardin GM, Dhent $M$, Pattyn P. Surgery for gastrointestinal endometriosis: indication and results. Acta Chir Belg . 2006.106:413-416 